\title{
Isatuximab: A Review of Its Use in Multiple Myeloma
}

\author{
James E. Frampton ${ }^{1}$ \\ Accepted: 6 July 2021 / Published online: 5 August 2021 \\ (c) Springer Nature 2021, corrected publication 2021
}

\begin{abstract}
Isatuximab (Sarclisa ${ }^{\circledR}$; isatuximab-irfc in the USA) is an anti-CD38 monoclonal antibody (mAb) approved for use in the treatment of adults with multiple myeloma (MM): in combination with pomalidomide and dexamethasone for those with relapsed and refractory MM (RRMM) who have received $\geq 2$ prior therapies, including lenalidomide and a proteasome inhibitor; and in combination with carfilzomib and dexamethasone for those with relapsed MM who have received $\geq 1$ prior therapy. In phase III studies, the addition of isatuximab to pomalidomide and dexamethasone significantly prolonged progression-free survival (PFS) and improved the depth of tumour response in patients with RRMM, as did the addition of isatuximab to carfilzomib and dexamethasone in patients with relapsed or refractory MM. Health-related quality of life was maintained when isatuximab was combined with these other therapies. Isatuximab-based combination therapies were generally well tolerated and demonstrated a manageable safety profile with no new safety signals. Although mature overall survival data are awaited, available evidence indicates that the combinations of isatuximab with pomalidomide and dexamethasone and isatuximab with carfilzomib and dexamethasone are important additional treatment options for RRMM and relapsed MM, respectively.
\end{abstract}

\section{Plain Language Summary}

The introduction of immunomodulatory drugs (IMiDs) protease inhibitors (PIs) and anti-CD38 monoclonal antibodies (mAbs) has improved survival in patients with multiple myeloma (MM) to the extent that this haematological malignancy is no longer viewed as an incurable disease, but rather as a manageable chronic condition characterized by multiple relapses and salvage therapies. Isatuximab (Sarclisa ${ }^{\circledR}$; isatuximab-irfc in the USA) is an anti-CD38 mAb approved for use in adult patients with relapsed/refractory MM (RRMM) and relapsed MM. Isatuximab prolonged progression-free survival (PFS) and increased the frequency and/or depth of tumour response when added to pomalidomide and dexamethasone in adults with RRMM who had received $\geq 2$ previous lines of treatment (ICARIA-MM trial), and when added to carfilzomib and dexamethasone in adults with relapsed or refractory MM who had received $\geq 1$ previous lines of treatment (IKEMA trial). Final overall survival (OS) data from both trials are awaited. Both isatuximab-based combination therapies had manageable safety profiles, with no new safety signals identified. Health-related quality of life was preserved. Currently available data indicate that the combinations of isatuximab with pomalidomide-dexamethasone and carfilzomib-dexamethasone are important additional treatment options for adults with RRMM and relapsed MM, respectively.

\section{Digital Features for this Adis Drug Evaluation can be found at} https://doi.org/10.6084/m9.figshare.14925378.

The manuscript was reviewed by: $S$. J. Harrison, Clinical Haematology, Peter MacCallum Cancer Centre and Royal Melbourne Hospital, Melbourne, VIC, Australia; J. Mikhael, Applied Cancer Research and Drug Discovery, Translational Genomics Research Institute, City of Hope Cancer Center, Phoenix, AZ, USA; J. Richter, Icahn School of Medicine at Mount Sinai, New York, NY, USA.

James E. Frampton

demail@springer.com

1 Springer Nature, Private Bag 65901, Mairangi Bay, Auckland 0754, New Zealand

\section{Introduction}

Multiple myeloma (MM) is a common haematological malignancy characterized by clonal expansion of transformed plasma cells in the bone marrow and increased production of monoclonal (M)-protein (nonfunctional intact immunoglobulins or immunoglobulin chains) $[1,2]$. It is associated with substantial morbidity and mortality due to end-organ damage [renal impairment (RI), hypercalcaemia, lytic bony lesions and anaemia], as well as complicating infections (the principal cause of death) arising both from the disease itself and its treatment [1-3].

Dramatic improvements in clinical outcomes, including survival, in patients with MM have accompanied the introduction 


\section{Isatuximab: clinical considerations in MM}

Anti-CD38 mAb

Given intravenously ( $250 \mathrm{~mL}$ fixed volume infusion)

Improves PFS and depth of tumour response when added to pomalidomide and dexamethasone (in RRMM) or carfilzomib and dexamethasone (in relapsed or refractory MM)

Manageable safety profile (common adverse events include infusion reactions and respiratory infections)

of autologous stem cell transplantation (SCT) and, subsequently, the advent of small molecule anti-myeloma agents, such as immunomodulatory drugs (IMiDs; e.g. thalidomide, lenalidomide and pomalidomide) and proteasome inhibitors (PIs; e.g. bortezomib, carfilzomib and ixazomib), that are used, mostly in three-drug regimens that include a steroid, as front-line therapies for newly diagnosed disease in both transplant-eligble and -ineligible patients [4-6]. However, while MM generally responds well to initial chemotherapy, often remitting completely, it remains an incurable condition for the majority of patients who experience serial relapses due to the emergence of (different) drug-resistant clones, and thereby become increasingly refractory to these standard treatment regimens [5, 7-10]. Patients double-refractory to an IMiD plus a PI have a particularly poor prognosis, with a median overall survival (OS) and progressionfree survival (PFS) of 9 and 5 months, respectively [11].

Against this background of need for additional novel treatment options, the more recent development of monoclonal antibodies (mAbs), including agents directed against SLAMF7 (elotuzumab) and CD38 [daratumumab and isatuximab (Sarclisa ${ }^{\circledR}$; isatuximab-irfc in the USA)], has therefore represented another transformative advance in the management of relapsed and/or refractory MM [7, 12, 13]. CD38 is a type II transmembrane protein that functions both as a receptor (impacting processes such as leukocyte migration and activation) and as a multifunctional ectoenzyme (modulating calcium signalling) [14, 15]. It is an attractive target for MM therapy, as it is expressed at high levels on normal plasma cells and MM cells, but at relatively low levels on normal lymphoid and myeloid cells and in some non-haemopoietic tissues $[14,15]$.

Isatuximab, an anti-CD38 IgG- $\mathrm{KAb}$, is approved in several countries worldwide, including those of the EU [16], as well as the USA [17] and Japan [18]: in combination with pomalidomide and dexamethasone for adults with relapsed/ refractory MM (RRMM) who have received $\geq 2$ prior therapies [16-18] (including lenalidomide and a PI [16, 17] and who are refractory to their last therapy [16]); and in combination with carfilzomib and dexamethasone for adults with relapsed $\mathrm{MM}$ who have received $\geq 1$ [16] or 1-3 [17] prior therapies. This article overviews data relevant to the use of isatuximab combination therapies in these settings.

\section{Pharmacodynamic Properties of Isatuximab}

Preclinical studies indicate that isatuximab induces the killing of CD38-bearing MM cells through multiple mechanisms of action that include fragment crystallizable $(\mathrm{Fc})$-dependent immune effector activities supplemented by $\mathrm{Fc}$-independent activities [19-22]. Fc-dependent activities include natural killer (NK) cell-mediated antibody-dependent cellular cytotoxicity (ADCC), antibody-dependent cellular phagocytosis (ADCP) and complement-dependent cytotoxicity; ADCC and ADCP are the predominant immune effector mechanisms for isatuximab in $\mathrm{MM}$ cells [21]. In vitro, the triggering of these cytotoxic functions by isatuximab was dependent on the expression level of CD38 [21]. In vivo, isatuximab prolonged survival in a human MM xenograft model in immunocompromised mice [20]. In terms of Fc-independent activities, isatuximab induced direct cytotoxicity against MM cells in vitro via caspase-dependent apoptosis and lysosome-mediated non-apoptotic cell killing [23].

As well as Fc-mediated effector functions and direct anti-tumour effects, isatuximab has demonstrated immunomodulatory effects in vitro that may contribute indirectly to the control of tumour growth in MM [21, 24, 25]. These include: direct activation of, and augmented MM cell lysis by, NK cells [21, 24]; augmented MM cell lysis by CD8+ cells [24]; and suppression of CD38+ regulatory T cells to mitigate an immunosuppressive tumour microenvironment by alleviating inhibition of NK and CD8+ cells [24, 26]. In addition, inhibition of CD38 ectoenzymatic activity by isatuximab decreases the production of immunosuppressive adenosine that likely alleviates the immunosuppressive microenvironment of the bone marrow niche in MM patients $[15,22]$. An 'in vivo vaccination' effect, whereby MM patients treated with isatuximab develop a T-cell adaptive immune response against $\mathrm{CD} 38$ as well as other MMassociated antigens, has also been described [27].

A decrease in absolute counts of total CD16+ and CD56+ NK cells, CD19+ B-cells, CD4+ T cells and regulatory T cells $(\mathrm{CD} 3+, \mathrm{CD} 4+, \mathrm{CD} 25+, \mathrm{CD} 127-)$ was seen in the peripheral blood of MM patients treated with isatuximab monotherapy [16].

By binding to CD38 expressed on the surface of red blood cells (RBCs), isatuximab may result in a false positive indirect antiglobulin test (indirect Coombs test) [16, 17]; based on the half-life of the drug, this interference may persist for $\approx 6$ months after the last infusion [16]. The indirect Coombs test was positive in $68 \%$ and $63 \%$ of the tested isatuximab-treated patients in the phase III studies in patients with RRMM (ICARIA-MM) or relapsed or refractory MM (IKEMA) (Sect. 4) [17, 28, 29]. 
As an $\operatorname{IgG} 1-\kappa \mathrm{mAb}$, isatuximab has the potential to be detected on both serum protein electrophoresis (SPE) and immunofixation (IFE) assays used to monitor endogenous M-protein; this interference can lead to inaccurate determination of depth of response according to International Myeloma Working Group (IMWG) criteria in some patients with IgG M-protein [16, 30]. In the phase III ICARIA-MM and IKEMA studies (Sect. 4), isatuximab interference with M-protein detection was explored using mass spectrometry (MS) to separate the isatuximab and M-protein signals [30, 31].

Preclinical studies suggest the antitumor effects of isatuximab are enhanced when it is combined with IMiDs and PIs [32]. Compared with isatuximab alone, isatuximab plus pomalidomide enhanced in vitro killing of CD38+ MM cells via both $\mathrm{ADCC}$ and direct anti-tumour effects to a greater extent than isatuximab plus lenalidomide [23]. The combination of isatuximab and pomalidomide also demonstrated enhanced in vivo activity compared with that of isatuximab or pomalidomide alone in a murine xenograft model utilizing the human MOLP-8 MM cell line [16, 17, 33]. The combination of isatuximab and carfilzomib showed additive antimyeloma effects in a murine xenograft model utilizing the human MM.S1 MM cell line [32].

\section{Pharmacokinetic Properties of Isatuximab}

Isatuximab demonstrates nonlinear pharmacokinetics with target-mediated drug disposition due to its binding to the CD38 receptor [16]. After intravenous (IV) administration of isatuximab at the approved dosage $(10 \mathrm{mg} / \mathrm{kg}$ weekly for 4 weeks and then every 2 weeks), the median time to reach steady state was 8 weeks $[16,17]$.

As a large protein, isatuximab is expected to be metabolized into small peptides by non-saturable proteolytic catabolism processes $[16,17]$. Isatuximab is eliminated by two parallel pathways: a nonlinear target-mediated pathway predominating at low concentrations and a nonspecific linear pathway predominating at higher concentrations [16]. Linear clearance of isatuximab predominates over the therapeutic plasma concentration range; clearance decreases over time by $50 \%$ to a steady state value of $9.55 \mathrm{~mL} / \mathrm{h}$ (0.229 L/day) [16, 34]. The terminal half-life of isatuximab is 28 days [16]. At steady state, $\geq 99 \%$ elimination of isatuximab from plasma was predicted to occur $\approx 2$ months after the last dose [17].

Based on a population pharmacokinetic analysis, age, gender and race, as well as RI and hepatic impairment, have no clinically meaningful effects on the pharmacokinetics of isatuximab and do not require any dose adjustment $[16,34]$. Isatuximab steady-state exposure does, however, decrease with increasing bodyweight, necessitating a bodyweightbased dosing approach [16, 34] (Sect. 6).

\section{Therapeutic Efficacy of Isatuximab}

The efficacy of adding isatuximab to a regimen consisting of either an IMiD (pomalidomide) or a PI (carfilzomib) plus dexamethasone is being evaluated in two ongoing, randomized, open-label, multinational, phase III studies in adults with RRMM (ICARIA-MM; Sect. 4.1) or relapsed or refractory MM (IKEMA; Sect. 4.2) (Fig. 1).

\subsection{In Combination with Pomalidomide and Dexamethasone}

To be eligible for inclusion in ICARIA-MM, adults with RRMM had to have measurable disease [M-protein $\geq 0.5 \mathrm{~g}$ / $\mathrm{dL}$ (serum) or $\geq 200 \mathrm{mg} / 24 \mathrm{~h}$ (urine)]; they also had to have received $\geq 2$ prior lines of therapy, including lenalidomide and a PI, and to be refractory to their last line of therapy (i.e. progressed on or within 60 days of the end of their last line of therapy). Ineligible patients included those previously treated with pomalidomide, those refractory to prior anti-CD38 mAb therapy, and those with primary refractory disease [16, 28].

Randomization to isatuximab-pomalidomide-dexamethasone $(n=154)$ or pomalidomide-dexamethasone alone $(n=$ 153) was stratified according to the number of prior lines of therapy ( $2-3$ vs $>3$ ) and age ( $<75$ vs $\geq 75$ years). All study medications were administered in 4-week (28-day) cycles. Based on the results of a phase $1 \mathrm{~b}$ feasibility study [35], patients in the isatuximab arm received IV isatuximab $10 \mathrm{mg} / \mathrm{kg}$ weekly during the first cycle and then every 2 weeks during all subsequent cycles; patients in both arms received oral pomalidomide $4 \mathrm{mg} /$ day for the first 3 weeks of each cycle and IV/oral dexamethasone $40 \mathrm{mg}$ ( $20 \mathrm{mg}$ if they were aged $\geq 75$ years) weekly [28]. All patients were premedicated prior to isatuximab infusions; the premedication regimen included the dexamethasone component of pomalidomide-dexamethasone. Treatment continued until disease progression (or beyond at investigator discretion), unacceptable toxicity or patient choice [28].

The primary endpoint was PFS per independent response committee (IRC); key secondary endpoints were the overall response rate (ORR) per IRC and OS. Efficacy analyses have been done in the intent-to-treat (ITT) population [28]. The primary analysis of PFS was performed after a median follow-up of 11.6 months (data cutoff: 11 October 2018) (Table 1) [28]; an updated analysis assessing longer-term outcomes (including OS) was performed after a median follow-up of 35.3 months (data cutoff: 1 October 2020) [36]. Some results are only available from the EU regulatory review of isatuximab [25] or from abstracts/posters [30, 36-40] (post hoc analyses, where stated [38-40]).

Demographic and disease characteristics at baseline were generally well balanced between the treatment groups [28]. Overall, patients had a median age of 67 years $(20 \%$ were aged $\geq 75$ years), $25 \%$ were at International Staging System 


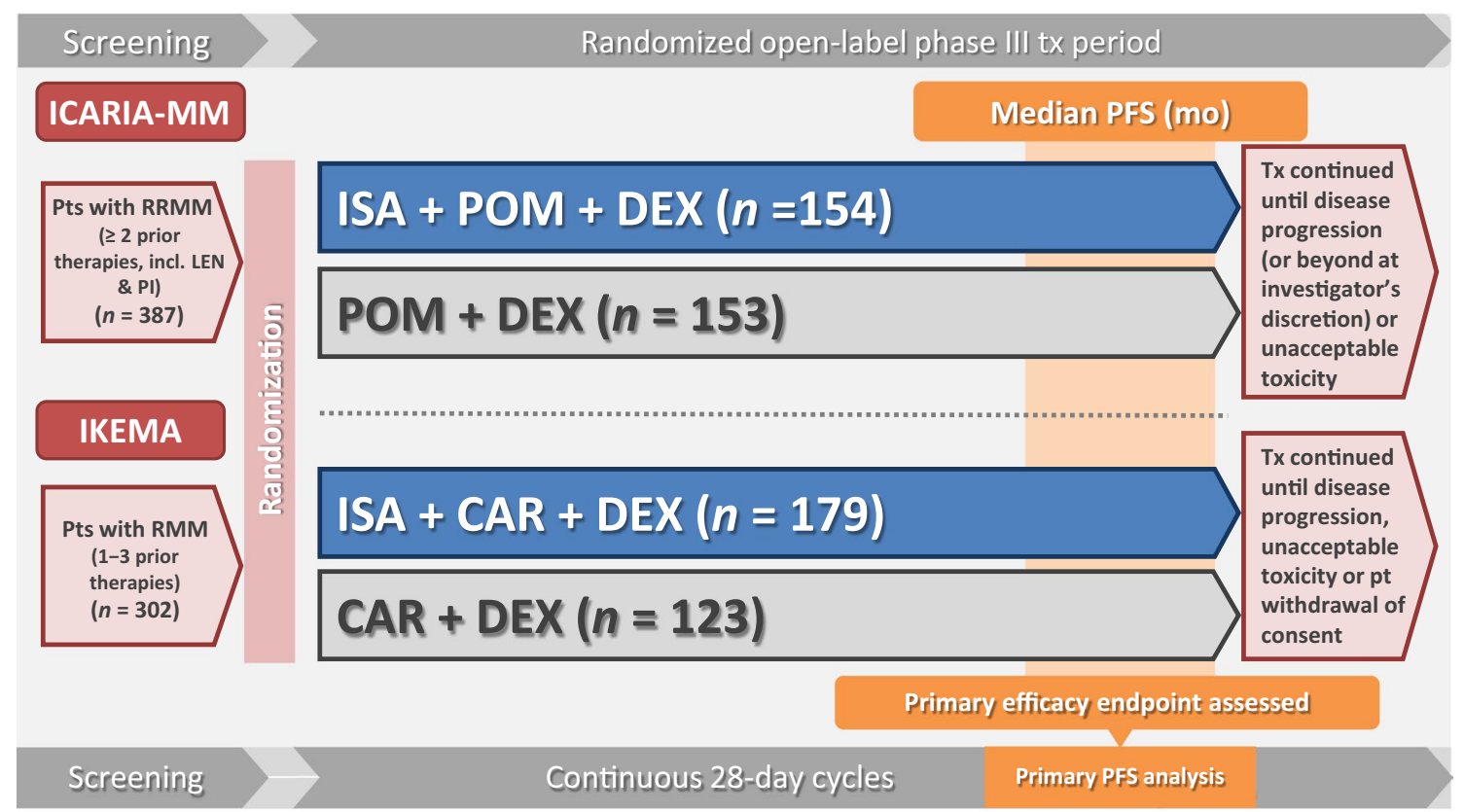

Fig. 1 Clinical trial design for the phase III ICARIA-MM and IKEMA trials, with key efficacy results in the animated figure (available online). Data are from the intent-to-treat population. $C A R$ carfilzomib, $D E X$ dexamethasone, ISA isatuximab, $L E N$ lenalidomide, $M M$

(ISS) disease stage III, and $28 \%$ were classified as frail (IMWG criteria) [28, 39]. Approximately a third (34\%) had RI [estimated glomerular filtration rate (eGFR) $<60 \mathrm{~mL} / \mathrm{min}$ per $1.73 \mathrm{~m}^{2}$ ]; $20 \%$ had high-risk cytogenetics [presence of $\geq 1$ of del( $(17 \mathrm{p}), \mathrm{t}(4 ; 14)$ or $\mathrm{t}(14 ; 16)]$. The median number of prior lines of therapy was 3 (range $2-11$ ); $56 \%$ of patients had received prior SCT; and $93 \%, 76 \%, 71 \%$ and $98 \%$ of patients were refractory to lenalidomide, a PI, lenalidomide plus a PI and their last line of therapy, respectively [28, 41].

The addition of isatuximab to pomalidomide-dexamethasone significantly improved PFS in patients with RRMM (Table 1); the risk of disease progression or all-cause death was reduced by $40 \%$ [hazard ratio (HR) $0.596,95 \%$ CI $0.436-0.814 ; p=0.001]$ at the time of the primary analysis [28]. A PFS benefit was seen with the addition of isatuximab to pomalidomide-dexamethasone in all prespecified subgroups, including patients aged $\geq 75$ years, those at ISS stage III, those with RI, those with high-risk cytogenetics, those with $2-3$ or $>3$ previous lines of therapy, those refractory to lenalidomide, those refractory to a PI, and those double-refractory to lenalidomide and a PI [28, 41, 42]. Moreover, in retrospective analyses, PFS benefits were seen with the addition of isatuximab to pomalidomide-dexamethasone in the following subgroups of patients: those with isolated gain of chromosome arm 1q21 genomic abnormality (1q21 gain; $\geq 3$ copies of 1q21); those with 1q21 gain, regardless of the presence of other high-risk cytogenetic multiple myeloma, mo months, $N R$ not reached, $P F S$ progression-free survival, $P I$ proteasome inhibitor, $P O M$ pomalidomide, $p t$ patient, $R R M M$ relapsed/refractory MM, $t x$ treatment.

abnormalities; those with isolated amplification of chromosome arm 1q21 genomic abnormality (1q21 amplification; $\geq 4$ copies of 1q21); and those with 1q21 amplification ( $\geq 4$ copies of 1q21), regardless of the presence of other highrisk cytogenetic abnormalities [42]. Improvements in PFS were also seen with the addition of isatuximab to pomalidomide-dexamethasone in the subgroups of patients classified as frail [39] and those with soft-tissue plasmacytomas [37].

Adding isatuximab to pomalidomide-dexamethasone significantly improved ORR (Table 1). Consistent with the ORR benefit seen in the overall population [odds ratio (OR) $2.795,95 \%$ CI 1.75-4.56; $p<0.0001$ ], ORRs were numerically higher with isatuximab-pomalidomide-dexamethasone than with pomalidomide-dexamethasone in all prespecified subgroups, including patients aged $\geq 75$ years, those with RI, those with high-risk cytogenetics, those with $2-3$ or $>3$ previous lines of therapy, those refractory to lenalidomide, and those double-refractory to lenalidomide and a PI [28, 41, 42]. ORRs were, respectively, $53.6 \%$ and $27.6 \%$ in isatuximabpomalidomide-dexamethasone and pomalidomide-dexamethasone recipients with isolated 1q21 gain (one-sided $p$ $=0.0116$ ) [42] and $52.1 \%$ and $34.2 \%$ in isatuximab-pomalidomide-dexamethasone and pomalidomide-dexamethasone recipients classified as frail $(p<0.05)$ [39].

Tumour responses were apparently faster and were more durable in patients receiving isatuximab combination therapy (Table 1). Tumour responses were also deeper, with the 
Table 1 Efficacy of isatuximab added to pomalidomide and dexamethasone or carfilzomib and dexamethasone in patients with relapsed and/or refractory multiple myeloma: intent-to-treat results from the phase III ICARIA-MM or IKEMA trials

\begin{tabular}{|c|c|c|c|c|}
\hline \multirow[t]{2}{*}{ Endpoint } & \multicolumn{2}{|l|}{ ICARIA-MM $^{\mathrm{a}}[16,28]$} & \multicolumn{2}{|l|}{$\operatorname{IKEMA}^{\mathrm{b}}[16,29]$} \\
\hline & ISA-Pd $(n=154)$ & $\operatorname{Pd}(n=153)$ & ISA-Kd $(n=179)$ & $\mathrm{Kd}(n=123)$ \\
\hline Median PFS ${ }^{\mathrm{c}, \mathrm{d}}$ (months) & $11.5^{*}$ & 6.5 & $\mathrm{NR}^{*}$ & 19.15 \\
\hline Median OS (months) & NR & NR & & \\
\hline ORR (\% pts) & $60 * *$ & 35 & 86.6 & 82.9 \\
\hline $\mathrm{sCR}^{\mathrm{d}}(\% \mathrm{pts})$ & 0 & $<1$ & & \\
\hline $\mathrm{CR}^{\mathrm{d}, \mathrm{e}}(\% \mathrm{pts})$ & 5 & 1 & 39.7 & 27.6 \\
\hline $\operatorname{VGPR}^{\mathrm{d}}(\% \mathrm{pts})$ & 27 & 7 & 33 & 28.5 \\
\hline$\geq \operatorname{VGPR}^{\mathrm{d}}(\%$ pts $)$ & $32 * *$ & 9 & $72.6 *{ }^{\mathrm{f}}$ & 56.1 \\
\hline $\mathrm{PR}^{\mathrm{d}}(\% \mathrm{pts})$ & 29 & 27 & 14 & 26.8 \\
\hline MRD-negative (\% pts) & 5.2 & 0 & $29.6 * \mathrm{f}$ & 13.0 \\
\hline Median TTR (months) & 1.2 & 1.9 & 1.1 & 1.1 \\
\hline Median DOR (months) & $13.3\left(<1^{\mathrm{g}}\right)$ & 11.1 & NR $[0.425(0.269-0.672)]^{\mathrm{g}}$ & NR \\
\hline Median TTP (months) & 12.7 & 7.75 & & \\
\hline Median TNT (months) & NR $[0.538(0.382-0.758)]^{\mathrm{g}}$ & 9.1 & NR $[0.566(0.380-0.841)]^{\mathrm{g}}$ & NR \\
\hline
\end{tabular}

Pts in ICARIA-MM had relapsed/refractory multiple myeloma; those in IKEMA had relapsed or refractory multiple myeloma

(s)CR (stringent) complete response, DOR duration of response, ISA isatuximab, $K d$ carfilzomib and dexamethasone, $M R D$ minimal residual disease, $N R$ not reached, $O R R$ overall response rate, $O S$ overall survival, $P d$ pomalidomide and dexamethasone, $P F S$ progression-free survival, $p t s$ patients, $P R$ partial response, $T N T$ time to next treatment, $T T P$ time to progression, $T T R$ time to first response, $V G P R$ very good partial response, $\geq V G P R$ VGPR or better

$* p \leq 0.0021, * * p<0.0001$ vs Pd (ICARIA-MM) or Kd (IKEMA)

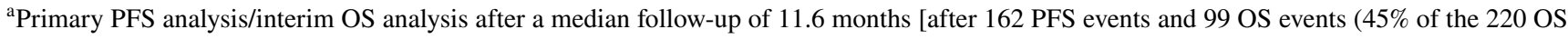
events planned for the final analysis)]

${ }^{\mathrm{b}}$ Interim PFS analysis after a median follow-up of 20.7 months [after 103 PFS events (65\% of the 159 PFS events planned for the final analysis)]

${ }^{c}$ Primary endpoint

${ }^{\mathrm{d}}$ Assessed by an independent response committee using International Myeloma Working Group response criteria

${ }^{\mathrm{e}} \mathrm{SCR}$ or CR (IKEMA)

${ }^{\mathrm{f}}$ Descriptive $p$ value

${ }^{\mathrm{g}}$ Hazard ratio (95\% CI) for ISA-Pd versus Pd (ICARIA-MM) or ISA-Kd versus Kd (IKEMA)

proportion of patients achieving a very good partial response or better ( $\geq$ VGPR) being nearly fourfold higher in the isatuximab-pomalidomide-dexamethasone group than the pomalidomide-dexamethasone group (Table 1) [28]. Moreover, MS analysis showed that the complete response (CR) rate in the isatuximab-pomalidomide-dexamethasone group was underestimated due to M-protein interference by isatuximab [30] (Sect. 2). Specifically, 11 of 22 evaluable isatuximab recipients with a near-CR (i.e. $<5 \%$ bone marrow plasma cells and negative SPE, but positive IFE) had M-protein values below the IFE sensitivity threshold $(0.25 \mathrm{~g} / \mathrm{dL})$. Ten of the 11 patients had $\operatorname{IgG}$ M-protein, confirming isatuximab interference predominantly affects patients with type IgG MM [30].

OS data were immature at the preplanned first interim analysis of this outcome, which was performed at the time of the primary PFS analysis [28]. Although median OS was not reached in either the isatuximab-pomalidomide-dexamethasone or pomalidomide-dexamethasone groups, the Kaplan-Meier survival curves showed an early and sustained separation that translated into a nonsignificant $31 \%$ decrease in the risk of death in patients receiving isatuximab combination therapy (HR 0.687, 95\% CI $0.461-1.023 ; p=0.0631$ ) [28]. At 1-year, OS rates were $72 \%$ in the isatuximab-pomalidomide-dexamethasone group versus $63 \%$ in the pomalidomide-dexamethasone group [25].

At the time of the updated analysis, an $\approx 7$-month improvement in median OS favouring isatuximab-pomalidomide-dexamethasone over pomalidomide-dexamethasone (24.6 months vs 17.7 months; HR 0.76 , 95\% CI $0.58-1.01$; one-sided $p=0.028$ ) was seen in the preplanned second interim analysis of this outcome [36]. The median time to next treatment was also increased significantly in the isatuximab-pomalidomide-dexamethasone group compared with the pomalidomide-dexamethasone group (15.5 months vs 8.9 months; HR $0.56,95 \%$ CI $0.42-0.74 ; p<0.0001$ ) [36].

Health-related quality of life (HRQOL), as assessed using the European Organization for Research and Treatment of Cancer Quality of Life questionnaire Core 30 (EORTC 
QLQ-C30), was preserved with the addition of isatuximab to pomalidomide-dexamethasone [40]. Over time, there were no clinically meaningful changes from baseline in the global health/quality of life (GHS/QOL), physical functioning (PF), pain and fatigue domains of the EORTC QLQ-C30 in either the isatuximab-pomalidomide-dexamethasone or pomalidomide-dexamethasone groups. In terms of the underlying trends, however, scores for all four of these key domains remained unchanged in isatuximab-pomalidomide-dexamethasone recipients but worsened in pomalidomide-dexamethasone recipients; the mean change per treatment cycle averaged across all cycles significantly favoured the former over the latter ( $p \leq 0.01$ for GHS/QOL and PF; $p \leq 0.05$ for pain and fatigue). Moreover, assessments of disease-relevant domains of the EORTC QLQ-C30 (GHS/QOL, PF, role functioning, pain and fatigue) and the EORTC myelomaspecific module (disease symptoms) suggested that HRQOL in the subgroups of patients with RI and those with $>3$ previous lines of therapy was maintained better in patients receiving isatuximab combination therapy [38].

The addition of isatuximab to pomalidomide-dexamethasone improved renal response rates in the subgroup of patients with RI [43]. Complete renal response $\left(C_{\text {renal }}\right.$; increase in eGFR from $<50 \mathrm{~mL} / \mathrm{min} / 1.73 \mathrm{~m}^{2}$ at baseline to $\geq 60 \mathrm{~mL} / \mathrm{min} / 1.73 \mathrm{~m}^{2}$ in $\geq 1$ post-baseline assessment) occurred in $72 \%$ of evaluable isatuximab-pomalidomide-dexamethasone recipients and $38 \%$ of evaluable pomalidomide-dexamethasone recipients; sustained $C_{\text {renal }}\left(C_{\text {renal }}\right.$ lasting $\geq 60$ days) occurred in $31 \%$ and $19 \%$ of patients [43].

\subsection{In Combination with Carfilzomib and Dexamethasone}

To be eligible for inclusion in IKEMA, adults with relapsed or refractory MM had to have measurable disease and to have received 1-3 prior lines of therapy, including IMiDs and PIs. Ineligible patients included those previously treated with carfilzomib, those refractory to prior anti-CD38 mAb therapy, and those with primary refractory disease [16, 29, 32].

Randomization to isatuximab-carfilzomib-dexamethasone $(n=179)$ or carfilzomib-dexamethasone alone $(n=123)$ was stratified according to the number of prior lines of therapy (1 vs $>1$ ) and disease stage (Revised-ISS I or II vs III vs not classified) [29, 32]. As in ICARIA-MM (Sect. 4.1), all study medications were administered in 4-week (28-day) cycles. Based partly on the results of an ongoing phase 1b study [44], patients in the isatuximab arm received IV isatuximab $10 \mathrm{mg} / \mathrm{kg}$ weekly for 4 weeks and then every 2 weeks; patients in both arms received IV carfilzomib twice weekly for the first 3 weeks of each cycle (20 $\mathrm{mg} / \mathrm{m}^{2}$ on days 1 and 2 of cycle $1 ; 56 \mathrm{mg} / \mathrm{m}^{2}$ on days $8,9,15$ and 16 of cycle 1 and thereafter) and dexamethasone $20 \mathrm{mg}$ twice weekly (on days 1, 2, 8, 9, 15, 16, 22 and 23 of each cycle; IV on days of isatuximab and/or carfilzomib infusions; orally on other days) [29, 32]. All patients were premedicated prior to isatuximab and/or carfilzomib infusions; the premedication regimen included the dexamethasone component of carfilzomib-dexamethasone [29, 32]. Treatment continued until disease progression, unacceptable toxicity or patient choice [29, 32].

The primary endpoint was PFS per IRC; key secondary endpoints were the ORR and $\geq$ VGPR per IRC, and OS. A preplanned interim analysis of PFS was performed after a median follow-up of 20.7 months (data cutoff: 7 February 2020) (Table 1) [16, 29]. Some results are only available from abstracts [31, 45-48] or the EU summary of product characteristics [16].

Baseline demographic and disease characteristics were generally well-balanced between the treatment groups [29]. Overall, patients had a median age of 64 years $(40 \%$ were aged $\geq 65$ years); $26 \%, 60 \%$ and $8 \%$ were at Revised-ISS stage I, II and III, respectively. Approximately a fifth (20\%) had RI, 24\% had highrisk cytogenetics, and $42 \%$ had $1 \mathrm{q} 21$ gain ( $\geq 3$ copies of $1 \mathrm{q} 21$ ). The median number of prior lines of therapy was 2 (range 1-4) $44 \%, 33 \%$ and $23 \%$ of patients had received 1,2 and $\geq 3$ prior lines of therapy respectively; and $61 \%$ had received $\geq 1$ prior SCT. Just over three-quarters $(78 \%)$ of patients had received prior IMiDs (including 43\% who had received prior lenalidomide); $90 \%$ of patients had received prior PIs; and $45 \%, 33 \%$, $33 \%$ and $54 \%$ of patients were refractory to an IMiD, lenalidomide, a PI and their last line of therapy, respectively [29].

The addition of isatuximab to carfilzomib-dexamethasone significantly improved PFS in patients with relapsed or refractory MM (Table 1); the risk of disease progression or all-cause death was reduced by $47 \%$ (HR $0.531,95 \% \mathrm{CI}$ $0.318-0.889 ; p=0.0013$ ) [16]. An isatuximab-related PFS advantage was seen across almost all prespecified subgroups, including patients aged $\geq 70$ years, those with RI, those with high-risk cytogenetics, those with 1q21 gain, those with 1 or $>1$ previous lines of therapy, those with prior IMiD or PI treatment, and those refractory to lenalidomide [29, 48, 49]. PFS benefits were also seen with the addition of isatuximab to carfilzomib-dexamethasone in the subgroups of patients with isolated 1q21 gain (HR 0.462, 95\% CI 0.219-0.972) and 1q21 gain combined with other high-risk cytogenetic abnormalities (HR 0.678, 95\% CI 0.299-1.537) [49].

Adding isatuximab to carfilzomib-dexamethasone was not associated with a significant increase in ORR, although there was a trend towards a more durable tumour response with isatuximab combination therapy at the time of this interim analysis (Table 1). Furthermore, deeper tumour responses were seen, with significantly more isatuximab-carfilzomib-dexamethasone recipients than carfilzomib-dexamethasone recipients achieving a $\geq$ VGPR (Table 1). Approximately twice as many patients in the isatuximab-carfilzomib-dexamethasone group than the carfilzomib-dexamethasone group who achieved $\geq$ VGPR also reached minimal residual disease (MRD)-negative status (41.4\% vs $22.9 \%$ ) [45]. Similarly, approximately twice 
as many patients in the isatuximab-carfilzomib-dexamethasone group than the carfilzomib-dexamethasone group who achieved CR also reached MRD-negative status $(20.1 \%$ vs $10.6 \%$; per ITT) [29, 31]. Furthermore, MS analysis showed the CR rate in the isatuximab-carfilzomib-dexamethasone group was underestimated due to isatuximab interference [31] (Sect. 2). Specifically, 11 of 27 evaluable isatuximab recipients with a near-CR or potential CR (i.e. serum remaining M-protein $\leq 0.5 \mathrm{~g} / \mathrm{dL}$ and positive IFE) had $<5 \%$ bone marrow plasma cells and M-protein values below the IFE sensitivity threshold; this indicated a potential adjusted CR rate of $45.8 \%$. Additionally, 7 of the 11 patients were MRD negative, suggesting the CR MRD-negative rate was also underestimated (potential adjusted rate of 24\%) [31]. Consistent with the overall population, the addition of isatuximab to carfilzomib-dexamethasone was associated with deeper responses in the subgroups of patients with RI [47], those with isolated 1q21 gain and 1q21 gain combined with other high-risk cytogenetic abnormalities [49], and those who had previously undergone SCT [46].

OS data were immature at the time of the interim efficacy analysis, with death rates of $17.3 \%$ and $20.3 \%$ in the isatuximab-carfilzomib-dexamethasone and carfilzomib-dexamethasone groups, respectively [45].

HRQOL, as assessed using the GHS/QOL domain of the EORTC QLQ-C30, was maintained with the addition of isatuximab to carfilzomib-dexamethasone [29].

$C_{\text {renal }}(52 \%$ vs $31 \%)$ and sustained $C_{\text {renal }}(32 \%$ vs $8 \%)$ rates were numerically higher in evaluable isatuximab-carfilzomib-dexamethasone recipients compared with evaluable carfilzomib-dexamethasone recipients [29].

\section{Tolerability of Isatuximab}

Isatuximab combination therapies were generally well tolerated and demonstrated a manageable tolerability profile with no new safety signals identified in the ICARIA-MM [28] and IKEMA [29] studies (Sect. 4).

Treatment-emergent adverse events (TEAEs) of any grade occurred in similar proportions of isatuximab-pomalidomide-dexamethasone and pomalidomide-dexamethasone recipients in ICARIA-MM (99\% vs 98\%) ( $n=152$ and 149, respectively) [25], as well as isatuximab-carfilzomib-dexamethasone and carfilzomib-dexamethasone recipients in IKEMA (97\% vs 96\%) [ $n=177$ and 122, respectively] [29]. The two isatuximab-based triplet regimens had largely similar safety profiles in terms of the (types of) most frequently reported adverse reactions (ARs), the most notable differences being neutropenia and hypertension, which were among the most common ARs in patients receiving pomalidomide-dexamethasone (with or without isatuximab) and carfilzomib-dexamethasone (with or without isatuximab), respectively [25,
29] (Fig. 2). Of note, the overall safety profile of isatuximab-pomalidomide-dexamethasone in the updated analysis remained unchanged from the earlier analysis [36].

The most frequent non-haematological ARs of any grade in isatuximab combination therapy recipients included infusion reactions (IRs), respiratory infections (upper respiratory tract infection, pneumonia and bronchitis) and dyspnoea [16, 25, 28, 29] (Fig. 2). All isatuximab recipients in ICARIA-MM and almost all isatuximab recipients in IKEMA (94\%) who had IRs experienced them during the first infusion of the drug; a small proportion of isatuximab recipients in ICARIA-MM also experienced them during their second and fourth infusions (2.0 and $1.3 \%$, respectively) $[16,17]$. Most IRs were mild or moderate (grade 1 or 2) in intensity [16, 25, 28, 29] (Fig. 2); all were reversible, resolving on the same day as the infusion in $98 \%$ and $74 \%$ of cases in ICARIA-MM and IKEMA, respectively $[16,17]$. Anaphylactic reactions occurred in $<1 \%$ of patients [17]. IRs led to the interruption of and the discontinuation of isatuximab in, respectively, $28.9 \%$ and $2.6 \%$ of patients who received the drug in ICARIA-MM, and $29.9 \%$ and $0.6 \%$ of patients who received the drug in IKEMA [16].

Grade $\geq 3$ TEAEs occurred more frequently in isatuximab-pomalidomide-dexamethasone than pomalidomide-dexamethasone recipients in ICARIA-MM ( $87 \%$ vs $71 \%$ ) [25], and in isatuximab-carfilzomib-dexamethasone than carfilzomib-dexamethasone recipients in IKEMA (77\% vs 67\%) [29]. Pneumonia was the most common grade $\geq 3$ non-haematological $\mathrm{AR}$ in isatuximab-pomalidomide-dexamethasone recipients (21.7\% and 3.3\% had grade 3 and 4 pneumonia, respectively, vs $16.1 \%$ and $2.7 \%$ of pomalidomide-dexamethasone recipients) [16]; it was the second most common grade $\geq 3$ AR in isatuximab-carfilzomib-dexamethasone recipients $(15.8 \%$ and $3.4 \%$ had grade 3 and 4 pneumonia, respectively, vs $10.7 \%$ and $2.5 \%$ of carfilzomib-dexamethasone recipients) [16] (Fig. 2). Overall, grade $\geq 3$ infections occurred in $43 \%$ of patients receiving isatuximab-pomalidomide-dexamethasone and 38\% of those receiving isatuximab-carfilzomib-dexamethasone. Infections leading to treatment discontinuation occurred in $2.6 \%$ of isatuximab-pomalidomide-dexamethasone recipients (vs 5.4\% of carfilzomib-dexamethasone recipients) and $2.8 \%$ of isatuximab-carfilzomib-dexamethasone recipients (vs $4.9 \%$ of carfilzomib-dexamethasone recipients); fatal infections occurred in $3.3 \%$ (vs $4.0 \%$ ) and $2.3 \%$ (vs $0.8 \%$ ) of patients, respectively [16].

In ICARIA-MM, serious TEAEs ( $62 \%$ vs $54 \%$ ) and serious treatment-related TEAEs (36\% vs $16 \%$ ) occurred more frequently in isatuximab-pomalidomide-dexamethasone than pomalidomide-dexamethasone recipients, albeit the exposureadjusted incidence rate of TEAEs was similar in the two groups (1.36 vs 1.30 per patient-year) [25]. In IKEMA, the incidences of serious TEAEs (59\% vs 57\%) and serious treatment-related TEAEs ( $25 \%$ vs $25 \%$ ) were similar in the isatuximab-carfilzomib-dexamethasone and carfilzomib-dexamethasone groups $[29,45]$. Moreover, in both studies, the addition of isatuximab 
did not result in an increase in ARs leading to definitive discontinuation of treatment $7.2 \%$ in isatuximab-pomalidomide-dexamethasone recipients vs $12.8 \%$ in pomalidomide-dexamethasone recipients in ICARIA-MM [25]; 8.5\% in isatuximab-carfilzomib-dexamethasone recipients vs $13.9 \%$ in carfilzomib-dexamethasone recipients in IKEMA [29, 45]) or ARs with a fatal outcome (7.2\% vs $8.7 \%$ in ICARIA-MM [25]; $3.4 \%$ vs $3.3 \%$ in IKEMA [29, 45]). The incidence of second primary malignancies (SPMs) was increased in patients receiving isatuximab combination therapies $[16,17]$. SPMs occurred in six patients (3.9\%) treated with isatuximab-pomalidomide-dexamethasone [vs one $(0.7 \%)$ treated with pomalidomide-dexamethasone] in ICARIA-MM, and $13(7.3 \%)$ treated with isatuximab-carfilzomib-dexamethasone [vs six (4.9\%) treated with carfilzomib-dexamethasone] in IKEMA [17]. The most common $(\geq 1 \%)$ SPMs in these phase III studies combined included skin cancers (4\% and $1.5 \%$ for regimens with and without isatuximab, respectively) and solid tumours other than skin cancer (1.8\% and $1.5 \%$, respectively). All patients with skin cancer continued isatuximab therapy after resection of the skin cancer [17].

Carfilzomib-associated cardiovascular adverse events in IKEMA included hypertension (Fig. 2) and cardiac failure events [29, 45]. Cardiac failure events of any grade were reported by $7.3 \%$ of isatuximab-carfilzomib-dexamethasone recipients versus $6.6 \%$ of carfilzomib-dexamethasone recipients; grade $\geq 3$ cardiac failure events were reported by $4.0 \%$ versus $4.1 \%$ of patients $[29,45]$. Serious and fatal cardiac failure, respectively, were seen in $4.0 \%$ and $1.1 \%$ of patients in the isatuximab-carfilzomib-dexamethasone group, and 3.3\% and $0 \%$ of patients in the carfilzomib-dexamethasone group [16].

With respect to haematological laboratory abnormalities, the incidences of grade $\geq 3$ neutropenia, thrombocytopenia and anaemia in ICARIA-MM were $85 \%, 31 \%$ and $32 \%$, respectively, with isatuximab-pomalidomide-dexamethasone versus $69 \%, 23 \%$ and $28 \%$, respectively, with pomalidomide-dexamethasone [16]; in IKEMA, they were 19\%, 30\% and 22\%, respectively, with isatuximab-carfilzomib-dexamethasone versus $7 \%, 24 \%$ and $20 \%$, respectively, with carfilzomib-dexamethasone [29, 45]. Haematological laboratory abnormalities leading to treatment discontinuation and/or dose modification and/or fulfilling a serious criterion were recorded as ARs [16]. Neutropenia was recorded as an AR in $46.7 \%$ of isatuximab-pomalidomide-dexamethasone recipients in ICARIA$\mathrm{MM}$ and in $4.5 \%$ of isatuximab-carfilzomib-dexamethasone recipients in IKEMA; the corresponding incidences of grade 3-4 neutropenia recorded as an AR were $45.4 \%$ and $4.0 \%$ [16, 29]. Grade $\geq 3$ febrile neutropenia occurred in $11.8 \%$ of patients treated with isatuximab-pomalidomide-dexamethasone and $1.1 \%$ of those treated with isatuximab-carfilzomib-dexamethasone; neutropenic infection (defined as infection with concurrent grade $\geq 3$ neutropenia) occurred in $25 \%$ and $1.7 \%$ of patients, respectively. Infections of the upper respiratory tract
(10\%), lower respiratory tract (9\%) and urinary tract (3\%) were the most frequent neutropenic infections in isatuximab-pomalidomide-dexamethasone recipients [16, 17].

The incidence of treatment-emergent anti-drug antibodies (ADAs) was $1.9 \%$ across nine clinical studies of isatuximab as monotherapy or in combination therapy in patients with MM, including ICARIA-MM and IKEMA $(n=1018)$ [16]. No effects of ADAs on the pharmacokinetics, safety or efficacy of isatuximab were observed [16].
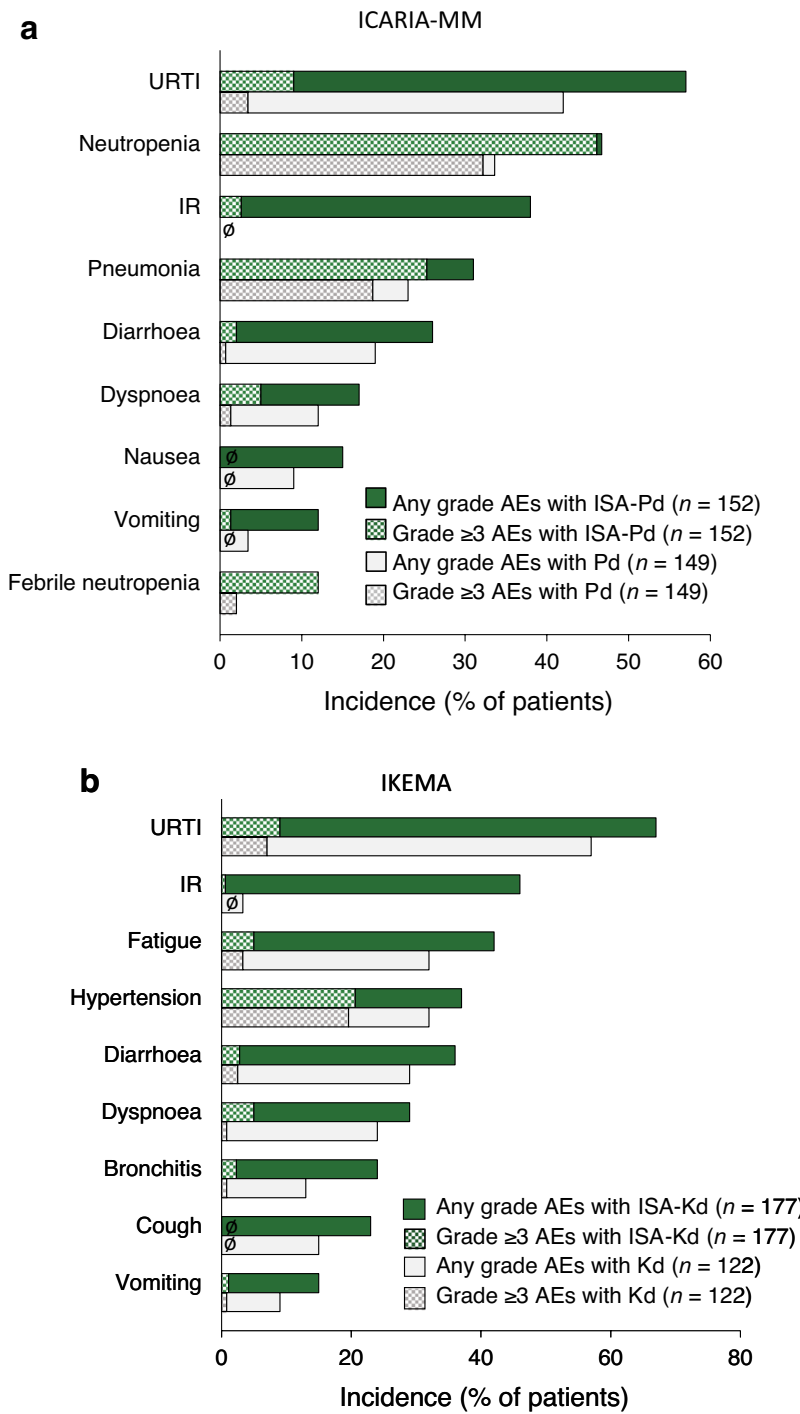

Fig. 2 Most common adverse events in patients receiving isatuximab added to a pomalidomide and dexamethasone (ICARIA-MM) and b carfilzomib and dexamethasone (IKEMA) [17, 25]. AEs adverse events, $I R$ infusion reaction, $I S A$ isatuximab, $K d$ carfilzomib and dexamethasone, $P d$ pomalidomide and dexamethasone, URTI upper respiratory tract infection. $\phi$ indicates zero incidence of any grade and/ or grade $\geq 3$ AEs 


\section{Dosage and Administration of Isatuximab}

When used in combination with pomalidomide and dexamethasone or carfilzomib and dexamethasone, the recommended dosage of isatuximab administered IV is $10 \mathrm{mg} /$ $\mathrm{kg}$ bodyweight on days $1,8,15$ and 22 (i.e. weekly) in the first 28-day treatment cycle and days 1 and 15 (i.e. every 2 weeks) in the second and all subsequent 28 -day cycles [16, 17]. Isatuximab is administered in a $250 \mathrm{~mL}$ fixed infusion volume, with initial infusion rates of 25,50 and $200 \mathrm{~mL} / \mathrm{h}$ for the first infusion, second infusion and all subsequent infusions, respectively. Incremental escalation of the infusion rate during the first and second infusions (to a maximum of 150 and $200 \mathrm{~mL} / \mathrm{h}$, respectively) should be considered only in the absence of IRs; administration adjustments (including, if necessary, permanent discontinuation) should be made if patients experience IRs [16].

Patients should receive premedication that also incorporates the dexamethasone component of pomalidomidedexamethasone or carfilzomib-dexamethasone 15-60 min prior to isatuximab infusion to reduce the risk and severity of IRs [16, 17]; those who do not experience an IR upon their first four infusions of isatuximab may have their need for subsequent premedication reconsidered [16].

Local prescribing information should be consulted for detailed information regarding the dosage and administration of isatuximab, recommended premedication agents, serological testing, use in special patient populations, and warnings and precautions.

\section{Place of Isatuximab in the Management of $\mathrm{MM}$}

The introduction of IMiDs, PIs and anti-CD38 mAbs with their attendant improvements in survival is shifting the perception of MM from being an incurable disease to being a manageable chronic disease characterized by multiple relapses and salvage therapies; the goal of treatment is to achieve disease control with acceptable toxicity and HRQOL [9].

The ongoing ICARIA-MM and IKEMA studies are the first phase III trials to evaluate and demonstrate the benefits of isatuximab combination therapies in patients with MM. In ICARIA-MM, the addition of isatuximab to pomalidomide-dexamethasone significantly improved PFS and ORR in patients with RRMM who had received $\geq 2$ previous lines of treatment (including lenalidomide and a PI) (Sect. 4.1), while in IKEMA, the addition of isatuximab to carfilzomib-dexamethasone significantly improved PFS in patients with relapsed or refractory MM who had received 1-3 prior lines of therapy (including IMiDs and PIs) (Sect. 4.2). In both studies, PFS benefits were seen across all patient subgroups, including those with poor prognoses (e.g. RI, high-risk cytogenetics). Tumour responses appeared more durable and, furthermore, were deeper with the addition of isatuximab to pomalidomide-dexamethasone or carfilzomib-dexamethasone; deeper tumour responses correlated with improved long-term outcomes [31, 50] (Sect. 4). Renal response rates in the RI subgroup were also improved in patients receiving isatuximab combination therapies (Sect. 4). OS data are immature in both ICARIA-MM and IKEMA, although there was a strong trend towards improvement with the addition of isatuximab in the former study, which is the more advanced of the two (Sect. 4). Mature OS data from both trials are awaited with considerable interest.

Isatuximab combination therapies were generally well tolerated and demonstrated a manageable safety profile with no new safety signals identifed in the ICARIA-MM [28] and IKEMA [29] studies (Sect. 5). Common ARs occurring with both triplet regimens included IRs, respiratory infections and dyspnoea, with neutropenia being the most common $\mathrm{AR}$ in patients receiving isatuximab-pomalidomide-dexamethasone (or pomalidomide-dexamethasone alone) and hypertension also being among the most common ARs in patients receiving isatuximab-carfilzomib-dexamethasone (or carfilzomib-dexamethasone alone). Most IRs occurred during the first infusion and were mild or moderate in intensity; all were reversible (Sect. 5). Premedication incorporating the dexamethasone component of pomalidomide-dexamethasone or carfilzomib-dexamethasone is administered prior to isatuximab infusion to reduce the risk and severity of IRs (Sect. 6); however, no post-infusion prophylaxis is mandated $[15,16]$. Isatuximab, particularly when combined with pomalidomide and dexamethasone, may cause neutropenia (Sect. 5); the use of colony-stimulating factors should be considered to mitigate the risk of this adverse event [16, 17]. Additionally, the incidences of infections and SPMs may be increased in patients receiving isatuximab combination therapies (Sect. 5); patients (including those with neutropenia) should be monitored for signs of infection and the occurrence of SPMs and managed accordingly $[16,17]$. Importantly, HRQOL is not adversely affected with the addition of isatuximab to pomalidomide-dexamethasone or carfilzomib-dexamethasone (Sect. 4).

Joint European Hematology Association (EHA) and European Society for Medical Oncology (ESMO) clinical practice guidelines for managing MM, including relapsed/refractory disease, have been published recently [51]. As a second-line therapy, isatuximab-carfilzomib-dexamethasone is recommended for patients who have been exposed or are refractory to lenalidomide; it can also be given in those who are refractory to bortezomib. As a third- or subsequentline therapy, isatuximab-pomalidomide-dexamethasone is a suitable option for patients who have failed $\geq 2$ lines of previous therapies, including lenalidomide and a PI, while isatuximab-carfilzomib-dexamethasone is a suitable option for those who have been exposed or are refractory to both bortezomib and lenalidomide (and who have not received an $\mathrm{mAb}$ ) [51]. Both isatuximab combination therapies have also been included in the latest National Comprehensive Cancer Network guidelines for MM [52]. As therapy for previously treated 
disease, isatuximab-pomalidomide-dexamethasone is a category 1 , preferred regimen for the treatment of RRMM (indicated for patients who have received $\geq 2$ prior therapies, including lenalidomide and a PI), while isatuximab-carfilzomib-dexamethasone is a category $2 \mathrm{~A}$, other recommended regimen [52].

Whereas isatuximab was administered in a weight-based infusion volume in ICARIA-MM and IKEMA, the approved method is administration in a $250 \mathrm{~mL}$ fixed infusion volume $[16,17]$ (Sect. 6). Use of the simplified fixed-volume infusion method was intended to reduce infusion times of isatuximab; the efficacy and safety of administering the drug in this approved manner has been confirmed in part 2 of the phase $1 \mathrm{~b}$ feasibility study of isatuximab-pomalidomide-dexamethasone [53]. Shorter duration infusion times offer greater convenience to patients; in the absence of IRs, the infusion rate of isatuximab can be progressively increased, such that the first, second and all subsequent infusions can be delivered in 200, 113 and 75 min, respectively [16, 17] (Sect. 6). Moreover, a subcutaneous (SC) formulation of isatuximab in combination with pomalidomide-dexamethasone is currently being evaluated (and compared with the IV formulation) in a phase Ib study in $\approx 46$ patients with RRMM (NCT04045795). Pending a successful outcome, the use of SC instead of IV isatuximab in combination with either pomalidomide-dexamethasone or carfilzomib-dexamethasone will increase the convenience of these regimens for patients.

Further long-term data on the efficacy and safety of isatuximab-pomalidomide-dexamethasone and isatuximab-carfilzomib-dexamethasone are required, as are studies confirming the effectiveness and tolerability of these triplet regimens in real-world settings. Regarding the former, the multinational IONA-MM trial (NCT04458831) is an ongoing, prospective, non-interventional, observational study of $\approx 1100$ patients with relapsed and/or refractory MM receiving isatuximab-pomalidomide-dexamethasone in routine clinical practice.

Comparative data in appropriately indicated patients with RRMM or relapsed MM are needed to help define the roles of the two isatuximab combination therapies relative to other regimens that may also be used in these settings. In particular, headto-head comparisons of isatuximab-based versus daratumumabbased regimens are keenly awaited. In the interim, median PFS and OS have been estimated to be, respectively, 5 and 8 months longer with isatuximab-pomalidomide-dexamethasone than with daratumumab-pomalidomide-dexamethasone, based on an indirect comparison of these two regimens that used data from the ICARIA-MM study for the former and data from a phase $1 \mathrm{~b}$ study in patients with relapsed and/or refractory MM for the latter [54]. As regards practical considerations, isatuximab, like daratumumab [55], may interfere with serological testing, as well as SPE and IFE (Sect. 2). It is, however, distinguishable from daratumumab, which requires prophylaxis both pre- and post-infusion [55]. Infusion times for IV isatuximab also compare favourably with those for IV daratumumab [15], albeit a SC formulation of the latter agent is already commercially available [55]. Furthermore, isatuximab binds to a CD38 epitope distinct from that targeted by daratumumab, and can be further differentiated from the latter on the basis of several mechanistic differences, including the ability to induce apoptosis of CD38-expressing cells directly (i.e. in the absence of cross-linking agents) [15]. Accordingly, the possibility that isatuximab can overcome resistance to daratumumab has begun to be explored [56]. Part B of the phase $1 \mathrm{~b}$ study enrolled seven patients who were refractory to daratumumab; three $(50 \%)$ of the six who were evaluable for response experienced a minimal response or better $(\geq \mathrm{MR})$ [53]. Moreover, seven (77\%) of nine heavily pretreated patients with RRMM who were refractory to daratumumab experienced $\mathrm{a} \geq \mathrm{MR}$ after receiving isatuximabpomalidomide-dexamethasone at the MD Anderson Cancer Center in the USA [57]. Larger, ideally controlled, studies of isatuximab in daratumumab-exposed or -refractory patients are warranted.

Pharmacoeconomic modelling studies based on indirect comparisons of efficacy suggest that, at 1 [58, 59] and 3 [58] (but not 5 [58]) years, there will be both clinical and-from a USA payer perspective-economic benefits favouring the use of IV isatuximab rather than IV daratumumb in combination with pomalidomide-dexamethasone [58] or carfilzomib-dexamethasone [59] to treat relapsed and/or refractory MM. Additional, longerterm comparisons of isatuximab-based versus daratumumab-based regimens in this, as well as a European, setting will be of interest, as will pharmacoeconomic studies comparing SC isatuximab (pending availability) with SC daratumumab.

In conclusion, although final OS data are awaited, the available evidence indicates that the combinations of isatuximab with pomalidomide-dexamethasone and carfilzomib-dexamethasone are important additional treatment options for RRMM and relapsed MM, respectively.

Data Selection Isatuximab: 254 records identified

Duplicates removed

Excluded during initial screening (e.g. press releases; news reports; not relevant drug/indication; preclinical study; reviews; case reports; not randomized trial)

Excluded during writing (e.g. reviews; duplicate data; small patient number; nonrandomized/phase I/II trials)

Cited efficacy/tolerability articles

Cited articles not efficacy/tolerability

Search Strategy: EMBASE, MEDLINE and PubMed from 1946 to present. Clinical trial registries/databases and websites were also searched for relevant data. Key words were isatuximab, Sarclisa, SAR650984, multiple myeloma. Records were limited to those in English language. Searches last updated 13 July 2021 
Supplementary Information The online version contains supplementary material available at https://doi.org/10.1007/s11523-021-00827-0.

Acknowledgements During the peer review process, the manufacturer of isatuximab was also offered an opportunity to review this article. Changes resulting from comments received were made on the basis of scientific and editorial merit.

\section{Declarations}

Funding The preparation of this review was not supported by any external funding.

Authorship and Conflict of Interest J. E. Frampton is a salaried employee of Adis International Ltd/Springer Nature, and declares no relevant conflicts of interest. All authors contributed to the review and are responsible for the article content.

Ethics approval, Consent to participate, Consent to publish, Availability of data and material, Code availability Not applicable.

Open Access This article is licensed under a Creative Commons Attribution-NonCommercial 4.0 International License, which permits any non-commercial use, sharing, adaptation, distribution and reproduction in any medium or format, as long as you give appropriate credit to the original author(s) and the source, provide a link to the Creative Commons licence, and indicate if changes were made. The images or other third party material in this article are included in the article's Creative Commons licence, unless indicated otherwise in a credit line to the material. If material is not included in the article's Creative Commons licence and your intended use is not permitted by statutory regulation or exceeds the permitted use, you will need to obtain permission directly from the copyright holder. To view a copy of this licence, visit http://creativecommons.org/licenses/by-nc/4.0/.

\section{References}

1. Gerecke C, Fuhrmann S, Strifler S, et al. The diagnosis and treatment of multiple myeloma. Dtsch Artzebl Int. 2016;113:470-6.

2. Cowan AJ, Allen C, Barac A, et al. Global burden of multiple myeloma; a systematic analysis for the global burden of disease study 2016. JAMA Oncol. 2018;4(9):1221-7.

3. Blimark C, Holmberg E, Mellqvist U-H, et al. Multiple myeloma and infections: a population-based study on 9253 multiple myeloma patients. Haematologica. 2015;100(1):107-13.

4. Moreau P, Attal M, Facon T. Frontline therapy of multiple myeloma. Blood. 2015;125(20):3076-84.

5. Pinto V, Bergantim R, Caires HR. Multiple myeloma: available therapies and causes of drug resistance. Cancers. 2020;12:407.

6. Rajkumar SV. Multiple myeloma: selection of initial chemotherapy for symptomatic disease. UpToDate. https://www.uptod ate.com/home. Accessed 18 Mar 2021.

7. Abramson HN. Monoclonal antibodies for the treatment of multiple myeloma: an update. Int J Mol Sci. 2018;19:3924.

8. Rajkimar SV. Multiple myeloma: 2020 update on diagnosis, riskstratification and management. Am J Haematol. 2020;95:548-67.

9. Bazarbachi AH, Al Hamed RA, Malard F, et al. Relapsed refractory multiple myeloma: a comprehensive overview. Leukemia. 2019;33:2343-57.

10. Bird SA, Boyd K. Multiple myeloma: an overview of management. Palliat Care Soc Pract. 2019;13:1-13.
11. Kumar SK, Lee JH, Lahuerta JJ, et al. Risk of progression and survival in multiple myeloma relapsing after therapy with IMiDs and bortezomib: a multicenter International Myeloma Working Group study. Leukemia. 2011;26:149-57.

12. Wudhikarna K, Wills B, Lesokhind AM. Monoclonal antibodies in multiple myeloma: current and emerging targets and mechanisms of action. Best Pract Res Clin Haematol. 2020;33(1):101143.

13. Chim CS, Kumar SK, Orlowski RZ, et al. Management of relapsed and refractory multiple myeloma: novel agents, antibodies, immunotherapies and beyond. Leukemia. 2018;32:252-62.

14. van de Donk NWCJ, Usmani SZ. CD38 Antibodies in multiple myeloma: mechanisms of action and modes of resistance. Front Immunol. 2018;9:2134.

15. Richardson PG. Isatuximab for the treatment of relapsed/refractory multiple myeloma. Expert Opin Biol Ther. 2020;12:1395-404.

16. Sanofi-Aventis. SARCLISA (isatuximab): EU summary of product characteristics. 2021. https://www.ema.europa.eu. Accessed 22 Apr 2021.

17. Sanofi-Aventis. SARCLISA $®$ (isatuximab-irfc): US prescribing information. 2021. https://www.fda.gov/drugs. Accessed 22 Apr 2021.

18. Sanofi Co., Ltd. Sarclisa intravenous infusion $100 \mathrm{mg} / \mathrm{Sarclisa}$ intravenous infusion $500 \mathrm{mg}$ : Japanese prescribing information. 2021. https://www.pmda.go.jp. Accessed 22 Apr 2021.

19. Deckert J, Wetzel MC, Bartle LM, et al. SAR650984, a novel humanized CD38-targeting antibody, demonstrates potent antitumor activity in models of multiple myeloma and other CD38+ hematologic malignancies. Clin Cancer Res. 2014;20(17):4574-83.

20. Moreno L, Perez C, Zabaleta A, et al. The mechanism of action of the anti-CD38 monoclonal antibody isatuximab in multiple myeloma. Clin Cancer Res. 2019;25(10):3176-87.

21. Zhu C, Song Z, Wang A, et al. Isatuximab acts through Fcdependent, independent, and direct pathways to kill multiple myeloma cells. Front Immunol. 2020;11(1771):1-19.

22. Martin TG, Corzo K, Chiron M, et al. Therapeutic opportunities with pharmacological inhibition of CD38 with isatuximab. Cells. 2019;8(12):1522

23. Jiang H, Acharya C, An G, et al. SAR650984 directly induces multiple myeloma cell death via lysosomal-associated and apoptotic pathways, which is further enhanced by pomalidomide. Leukemia. 2016;30(2):399-408.

24. Feng $\mathrm{X}$, Zhang L, Acharya $\mathrm{C}$, et al. Targeting CD38 suppresses induction and function of $\mathrm{T}$ regulatory cells to mitigate immunosuppression in multiple myeloma. Clin Cancer Res. 2017;23(15):4290-300.

25. European Medicines Agency. Sarclisa (isatuximab): assessment report. https://www.ema.europa.eu. Accessed 28 Jan 2021.

26. Tai Y-T, Anderson KC. Targeting CD38 alleviates tumor-induced immunosuppression. Oncotarget. 2017;8(68):112166-7.

27. Atanackovic D, Yousef S, Shorter C, et al. In vivo vaccination effect in multiple myeloma patients treated with the monoclonal antibody isatuximab. Leukemia. 2020;34(1):317-21.

28. Attal M, Richardson PG, Rajkumar SV, et al. Isatuximab plus pomalidomide and low-dose dexamethasone versus pomalidomide and low-dose dexamethasone in patients with relapsed and refractory multiple myeloma (ICARIA-MM): a randomised, multicentre, open-label, phase 3 study. Lancet. 2019;394(10214):2096-107.

29. Moreau P, Dimopoulos M-A, Mikhael J, et al. Isatuximab, carfilzomib, and dexamethasone in relapsed multiple myeloma (IKEMA): a multicentre, open-label, randomised phase 3 trial. Lancet. 2021;397(10292):2361-71.

30. Finn $\mathrm{G}$, Mace $\mathrm{S}$, Campana $\mathrm{F}$, et al. Evaluating isatuximab interference with monoclonal protein detection by immuno-capture and liquid chromatography coupled to high resolution mass spectrometry in the pivotal phase 3 multiple myeloma trial, ICARIA-MM [abstract no. 3143]. In: American Society of Hematology 61st Annual Meeting. 2019. 
31. Martin T, Mikhael J, Hajek R, et al. Depth of response and response kinetics of isatuximab plus carfilzomib and dexamethasone in relapsed multiple myeloma: Ikema interim analysis. Blood. 2020;136(Suppl 1):7-8.

32. Moreau P, Dimopoulos MA, Yong K, et al. Isatuximab plus carfilzomib/dexamethasone versus carfilzomib/dexamethasone in patients with relapsed/refractory multiple myeloma: IKEMA phase III study design. Future Oncol. 2020;16(2):4347-58.

33. US FDA Center for Drug Evaluation and Research. SARCLISA® (isatuximab-irfc): multi-discipline review. https://www.fda.gov/ drugs. Accessed 11 Mar 2021.

34. Fau JB, El-Cheikh R, Brillac C, et al. Drug-disease interaction and time-dependent population pharmacokinetics of isatuximab in relapsed/refractory multiple myeloma patients. CPT Pharmacomet Syst Pharmacol. 2020;9(11):649-58

35. Mikhael J, Richardson P, Usmani SZ, et al. A phase 1b study of isatuximab plus pomalidomide/dexamethasone in relapsed/refractory multiple myeloma. Blood. 2019;134(2):123-33.

36. Richardson PG, Perrot A, San-Miguel JF, et al. Updates from ICARIA-MM, a phase 3 study of isatuximab (Isa) plus pomalidomide and low-dose dexamethasone (Pd) versus Pd in relapsed and refractory multiple myeloma (RRMM) [abstract no. 8017]. J Clin Oncol. 2021;39(Suppl 15):8017.

37. Beksac M, Richardson PG, Unal A, et al. Isatuximab plus pomalidomide and dexamethasone in patients with relapsed/refractory multiple myeloma and soft-tissue plasmacytomas: ICARIAf-MM subgroup analysis [abstract no. EP978]. HemaSphere. 2020;4(Suppl 1):446.

38. Dimopoulos M, Campana F, Bury DP, et al. Health-related quality of life in heavily pretreated and renally impaired patients with relapsed/refractory multiple myeloma receiving isatuximab plus pomalidomide and dexamethasone: ICARIA-MM study [abstract no. EP1028]. HemaSphere. 2020;4(Suppl 1):473-4.

39. Schjesvold F, Bringhen $S$, Richardson $P$, et al. Isatuximab plus pomalidomide and dexamethasone in frail patients with relapsed/refractory multiple myeloma: Icaria-MM subgroup analysis [abstract no. 1411]. In: American Society of Hematology 62nd Annual Meeting. 2020.

40. Houghton K, Dimopoulos MA, Lin P, et al. Health-related quality of life in patients with relapsed/refractory multiple myeloma treated with isatuximab plus pomalidomide and dexamethasone: ICARIA-MM study. Blood. 2019;134(Suppl 1):1850.

41. Bringhen $\mathrm{S}$, Pour L, Vorobyev V, et al. Isatuximab plus pomalidomide and dexamethasone in patients with relapsed/refractory multiple myeloma according to prior lines of treatment and refractory status: ICARIA-MM subgroup analysis. Leuk Res. 2021;104:106576.

42. Harrison SJ, Perrot A, Alegre A, et al. Subgroup analysis of ICARIAMM study in relapsed/refractory multiple myeloma patients with highrisk cytogenetics. Br J Haematol. 2021;194(1):120-31.

43. Dimopoulos MA, Leleu X, Moreau P, et al. Isatuximab plus pomalidomide and dexamethasone in relapsed/refractory multiple myeloma patients with renal impairment: ICARIA-MM subgroup analysis. Leukemia. 2021;35:562-72.

44. Chari A, Richter JR, Shah N, et al. Phase I-b study of isatuximab + carfilzomib in relapsed and refractory multiple myeloma (RRMM) [abstract no. 8014]. J Clin Oncol. 2018;36(15 Suppl):8014.

45. Moreau P, Dimopoulos MA, Mikhael J, et al. Isatuximab plus carfilzomib and dexamethasone vs carfilzomib and dexamethasone in relapsed/ refractory multiple myeloma (Ikema): interim analysis of a phase 3, randomized, open-label study [abstract no. LB2603 plus oral presentation]. In: European Haematology Association 25th Congress. 2020.

46. Martin TG, Capra M, Mohty M, et al. Isatuximab plus carfilzomib and dexamethasone versus carfilzomib and dexamethasone in relapsed multiple myeloma patients who previously underwent transplantation: Ikema subgroup analysis [abstract no. 66]. Transpl Cell Ther. 2021;27(3 Suppl):S65.

47. Capra M, Martin T, Moreau P, et al. Isatuximab plus carfilzomib and dexamethasone versus carfilzomib and dexamethasone in relapsed multiple myeloma patients with renal impairment: Ikema subgroup analysis [abstract]. Blood. 2020;136(Suppl 1):46-7.

48. Facon T, Moreau P, Martin TG, et al. Isatuximab plus carfilzomib and dexamethasone versus carfilzomib and dexamethasone in elderly patients with relapsed multiple myeloma: IKEMA subgroup analysis [abstract no. 8026]. J Clin Oncol. 2021;39(Suppl 15):8026.

49. Spicka I, Moreau P, Martin TG, et al. Isatuximab plus carfilzomib and dexamethasone in relapsed multiple myeloma patients with high-risk cytogenetics: IKEMA subgroup analysis [abstract no. 8042]. J Clin Oncol. 2021;39(Suppl 15):8042.

50. Hulin C, Richardson PG, Attal M, et al. Depth of response and response kinetics in the ICARIA-MM study of isatuximab with pomalidomide/dexamethasone in relapsed/refractory multiple myeloma [abstract no. 3185]. Blood. 2019;134(Suppl 1):3185.

51. Dimopoulos MA, Moreau P, Terpos E, et al. Multiple myeloma: EHA-ESMO Clinical Practice Guidelines for diagnosis, treatment and follow-up. Ann Oncol. 2021;32(3):309-22.

52. National Comprehensive Cancer Network. NCCN guidelines version 7 . 2021. Multiple myeloma. https://www.nccn.org/. Accessed 6 Jul 2021.

53. Usmani SZ, Karanes C, Bensinger WI, et al. Final results of a phase $1 \mathrm{~b}$ study of isatuximab short-duration fixedvolume infusion combination therapy for relapsed/refractory multiple myeloma. Leukemia. 2021. https://doi.org/10.1038/s41375-021-01262-w.

54. Chari A, Suvannasankha A, Fay JW, et al. Daratumumab plus pomalidomide and dexamethasone in relapsed and/or refractory multiple myeloma. Blood. 2017;130(8):974-81.

55. Janssen-Cilag International NV. Darzalex (daratumumab): EU summary of product characteristics. 2021. https://www.ema. europa.eu. Accessed 22 Apr 2021.

56. Mikhael J, Belhadj-Merzoug K, Hulin C, et al. A phase 2 study of isatuximab monotherapy in patients with multiple myeloma who are refractory to daratumumab. Blood Cancer J. 2021;11:89.

57. Becnel M, Horowitz S, Thomas SK, et al. Descriptive analysis of isatuximab use following prior daratumumab in patients with relapsed/refractory multiple myeloma [abstract no. 1371]. In: American Society of Hematology 62nd Annual Meeting. 2020.

58. Alrawashdh N, Almutairi A, McBride A, et al. Economic evaluation of daratumumab and pomalidomide and dexamethasone versus isatuximab and pomalidomide and dexamethasone for patients with relapsed or refractory multiple myeloma [abstract no. 3419]. In: American Society of Hematology 62nd Annual Meeting. 2020.

59. Alrawashdh N, Choi B, Obeng-Kusi M, et al. Economic evaluation of 6 and 12 month $(\mathrm{m})$ treatment with isatuximab and carfilzomib and dexamethasone (IKd) versus daratumumab and carfilzomib and dexamethasone (DKd) in patients with relapsed or refractory multiple myeloma (RRMM) [abstract no. e20010]. J Clin Oncol. 2021;39(Suppl 15):e20010. 